\title{
educação

\section{Autoeficácia formativa e envolvimento nos processos de aprendizagem de estudantes portugueses maiores de 23 anos}

\author{
RitA BARROS \\ Instituto Piaget de Vila Nova de Gaia, Portugal \\ Angélica Monteiro ${ }^{\text {II }}$ \\ Universidade do Porto, Portugal \\ Cláudia Sousa III \\ Universidade Lusófona do Porto, Portugal
}

\begin{abstract}
A aprovação do regime de acesso ao Ensino Superior pela via dos "maiores de 23" constitui um dos principais desafios com que se deparam as Instituições de Ensino Superior (IES) portuguesas, que se traduz na necessidade de construção de respostas educativas tendo em conta a heterogeneidade do seu público. O estudo exploratório aqui apresentado tem por objetivo retratar a autoperceção destes estudantes acerca da sua eficácia formativa e o seu envolvimento académico, em função da idade de ingresso no Ensino Superior, assim como explorar a relação entre estas autoperceções, suas dimensões e idade de ingresso no Ensino Superior. Foram recolhidos dados junto de 55 estudantes de duas IES privadas, através de inquérito por questionário, os quais foram sujeitos a análises descritivas e de regressão linear. Os resultados mostram que é possível diferenciar dois grupos de estudantes em função da idade, até aos 26 anos e mais, sendo que este último se destaca pelo maior envolvimento académico e pela maior autoperceção de eficácia formativa. A discussão destes resultados, à luz dos referenciais teóricos do desenvolvimento da idade adulta, assume particular relevância para a promoção da equidade de acesso e ingresso no Ensino Superior.
\end{abstract}

Palavras-chave: Ensino Superior; Autoeficácia formativa; Envolvimento académico

\section{IN T R O D U Ç Ã O}

O conjunto de mudanças decorrentes da globalização, dos avanços tecnológicos e da pós-modernidade gera novos desafios ao Ensino Superior. Em resposta, os governos europeus desenvolvem iniciativas, de que é o exemplo o "Processo de Bolonha", desencadeado por uma Declaração Conjunta assinada em junho de 1999, e que tem como objetivo a criação de um Espaço Europeu de Ensino Superior coeso e competitivo. Neste contexto, são desenvolvidas regulações nacionais, por parte dos Estados-membros, 
que contribuam para gerir as demandas do ponto de vista académico, social, económico e, também, para dar resposta às exigências europeias de qualidade, que se refletem através das estatísticas. Alguns dos resultados incluem o aumento das qualificações da população portuguesa, o reconhecimento da experiência profissional, uma maior mobilidade e flexibilidade dos percursos de aprendizagem, e resultam numa maior heterogeneidade de públicos que frequentam o Ensino Superior.

Instituído pelo Decreto-Lei n. ${ }^{\circ}$ 64/2006, o regime de acesso ao Ensino Superior por maiores de 23 (M23) no contexto português visa a "promoção de igualdade de oportunidades no acesso a este grau de ensino, atraindo novos públicos, numa lógica de aprendizagem ao longo de toda a vida" (Decreto-Lei n. ${ }^{\circ}$ 64/2006, p. 2054). Este regime tem como destinatários estudantes M23 que demonstrem ter a capacidade para a frequência de um curso de licenciatura num estabelecimento de Ensino Superior. Cabe ao órgão legal e estatutariamente competente de cada Instituição de Ensino Superior (IES) aprovar o regulamento das provas destinadas a avaliar a referida capacidade para a frequência do Ensino Superior, sendo obrigatório integrar, no processo de avaliação, a apreciação do currículo escolar e profissional, a avaliação das motivações do candidato e a realização de provas de avaliação dos conhecimentos e competências considerados indispensáveis ao ingresso e progressão no curso.

Segundo Brás, Jezine, Fonseca, e Gonçalvez (2012), “o acesso à universidade aos maiores de 23 anos vem tentar repor uma oportunidade de frequentar a universidade que, por circunstâncias variadas da vida, lhes foi negada" (p. 164). Este facto é particularmente relevante em Portugal, onde apenas $24,3 \%$ da população residente no Continente com idade entre 25 e 64 anos completou o Ensino Superior completo $^{1}$. Segundo os dados da Direção-Geral de Estatísticas da Educação e Ciência (DGEEC) - relativos aos estudantes inscritos no $1 .^{\circ}$ ano, pela primeira vez, no ano letivo 2016/17-, num universo de 45.460 estudantes, cerca de $2.939(6,5 \%)$ ingressaram através da realização de prova para os M23. Este estudante "nãotradicional”, em geral, está inserido no mundo do trabalho, vive autonomamente da sua família de origem ou tem responsabilidades parentais, não tendo concluído o Ensino Secundário (Baptista, Bessa, \& Tavares, 2009; Monteiro, Barros, \& Moreira, 2015; Soares, Almeida, \& Ferreira, 2010). Este estudante, entendido como aquele que pertence a um grupo minoritário no Ensino Superior, está limitado na sua participação por razões estruturais, nas quais se inclui, naturalmente, a idade (estudantes mais maduros), o género (sobretudo, mulheres), a situação profissional (trabalhadores), o facto de serem os primeiros das suas famílias a frequentar o Ensino Superior, a pertença a populações migrantes, entre outras (Almeida, Quintas, \& Gonçalves, 2016).

Esta realidade, ainda que com pouca representatividade (Soares et al., 2010), tem como efeitos um aumento da faixa etária dos estudantes, uma maior democratização no acesso e o aumento da diversidade do público que frequenta o Ensino Superior, obrigando a que as instituições também mudem no sentido de se adequarem às necessidades que decorrem deste processo. De acordo com dados disponibilizados pela Direção-Geral do Ensino Superior (DGES, 2018), entre 2014 e 2017, o número de estudantes que entrou nas IES por esta via diminuiu $2,7 \%$, sendo que este decréscimo atingiu sobretudo as instituições

${ }^{1}$ Fonte: Instituto Nacional de Estatística. Período de referência dos dados: 2017. Consultado em novembro 2, 2018, em https:// www.ine.pt/xurl/indx/0006400/PT 
privadas, com uma queda de $51,9 \%$ do número de inscritos por esta via. Acresce o facto de que, segundo Engrácia e Baptista (2018), a taxa de abandono dos estudantes que ingressaram no Ensino Superior por este contingente é de cerca de $50 \%$, contra $21 \%$ dos que entraram pelo regime geral de acesso, o que implica a necessidade de haver mais estudos que permitam conhecer melhor estes estudantes e o seu envolvimento académico.

Do ponto de vista teórico, de acordo com Levinson (1977), no início da vida adulta (dos 22 aos 28 anos), são redefinidos e consolidados os alicerces da vida adulta, através das tarefas de desenvolvimento de exploração de possibilidades e de uma estrutura de vida estável. O jovem adulto deve estabelecer uma estrutura de vida que providencie uma ligação entre o Eu e a sociedade adulta, construindo o seu novo lar, definindo-se como um Adulto Jovem e lidando com as suas escolhas iniciais no que diz respeito à sua profissão, relações amorosas, estilo de vida e valores. Segundo o mesmo autor, uma tarefa fundamental para o jovem adulto é a de arquitetar uma profissão, tarefa que se alonga para além da fase da Idade Adulta Jovem, aplicando-se quer aos adultos que estabeleceram um compromisso bastante cedo com uma profissão, quer aos que permanecem indecisos, quer ainda aos que fazem grandes mudanças entre os 20 e os 30 anos.

Arnett (2000) aponta a inconsistência de se abordar a idade adulta jovem de uma forma una (dos 18 aos 30 anos), dado que a idade adulta jovem envolve períodos muito distintos. O termo 'jovem adulto' aplica-se aos indivíduos por volta dos 30 anos e, para a maioria das pessoas, a idade adulta jovem intensifica-se no fim dos vintes e é atingida pelos 30 anos. Na transição dos 30 anos (dos 28 aos 33 anos), os sujeitos podem fazer novas escolhas ou reafirmar as antigas. A consciência de modificação da vida pode levar a um sentido de urgência, o que pressupõe tomadas de decisão mais vinculativas, como por exemplo retomar os estudos. A maturidade é um critério de natureza psicológica que se reporta à capacidade de assumir responsabilidades, de raciocinar e de tomar decisões lógicas, de lidar com frustrações e superá-las e de aceitar os papéis definidos pela sociedade. A maturidade implica autonomia e autogestão, maior autoconhecimento, mais experiência, mais confiança e maior foco, requisitos essenciais no percurso académico do estudante do Ensino Superior no século XXI.

Desde 2007 que o Conselho da União Europeia declarou a pertinência da criação de oportunidades de Aprendizagem ao Longo da Vida nas Instituições de Ensino Superior, bem como o alargamento deste nível de ensino "a todos". Face a este novo cenário, as IES confrontam-se com o desafio de dar respostas às necessidades destes estudantes. Algumas destas respostas são essencialmente de natureza administrativa, como é o caso da disponibilização da formação em horário diurno e pós-laboral, ou da disponibilização de informação e aviso de alterações, horários de aulas, exames e trabalhos a realizar (Pereira, 2008). Também no que diz respeito aos candidatos com perfil compatível com os estudantes maiores de 23 anos, o alargamento e flexibilização do acesso ao Ensino Superior traduz-se na responsabilização das instituições na seleção dos candidatos, tomando como critério a sua experiência profissional, conforme a Lei 49/2005, e na avaliação da "capacidade para a frequência" de um curso de licenciatura, de acordo com a regulamentação das provas, com incidência exclusiva nas áreas de conhecimento diretamente relevantes para o curso, tal como previsto no DecretoLei n. ${ }^{\circ}$ 64/2006 (Pires, 2008). Para além das provas teóricas ou práticas de avaliação 
de conhecimentos e de competências considerados indispensáveis ao acesso e progressão no curso, são componentes obrigatórias da avaliação a apreciação do currículo académico e profissional do candidato e a avaliação das suas motivações, normalmente através de entrevista. Mas o alargamento do acesso e da participação destes novos estudantes não se restringe ao acionar de novos mecanismos facilitadores da entrada. A sua progressão e sucesso académico estão dependentes de aspetos organizacionais e pedagógico-didáticos que caracterizam as IES e o seu projeto educativo.

Do ponto de vista pedagógico-didático, a possibilidade de formação em e-learning, b-learning, ou simplesmente o recurso a Sistemas de Gestão de Aprendizagem para reposição de materiais, desenvolvimento de atividades ou interação com pares e professores constituem uma vantagem inegável para estes estudantes. Em todo o caso, os estudantes mais velhos podem beneficiar de apoio à utilização de Tecnologias de Informação e Comunicação, o que constitui mais um desafio para as IES (Pereira, 2008). Ventura (2009) aposta ainda nas intervenções de natureza multidisciplinar e transversal, articuladoras das políticas educativas, organização curricular, apoio a estudantes e formação de professores. Estes dois últimos aspetos são também sublinhados por Soares (2016), nomeadamente na implementação de tutorias para orientação e acompanhamento destes estudantes. Silva e Nascimento $(2010,2012)$ colocam a tónica nos projetos de investigação-ação subjacentes aos apoios na transição destes estudantes para o Ensino Superior.

As respostas acima referidas vão ao encontro de alguns dos fatores de influência para a participação dos estudantes adultos no Ensino Superior apresentadas por Davies, Osborne, e Williams (2002): (i) as políticas e práticas das Instituições de Ensino Superior flexibilização da oferta, horários pós-laborais, mudança nos curricula, ...; (ii) as políticas educativas e apoios financeiros - o que engloba as estratégias educativas de alargamento do acesso, e elementos como propinas, bolsas, empréstimos, benefícios fiscais, entre outros; a par do (iii) contexto económico e do mercado de trabalho; e da (iv) situação e circunstâncias individuais.

$\mathrm{O}$ acesso ao Ensino Superior por estudantes "não-tradicionais" tem vindo também a ser regulamentado noutros países. No caso espanhol, a legislação estabelece a normativa dos procedimentos de admissão aos cursos universitários oficiais de graduação (El Real Decreto 412/2014), prevendo a prova de acesso para os maiores de 25 anos, assim como para os maiores de 45 anos, o que vai ao encontro das especificidades desenvolvimentais das fases da idade adulta. Em contrapartida, no Reino Unido, algumas universidades definem as condições especiais de acesso para os intitulados estudantes maduros ("mature students"), tendo como requisito de idade os 21 anos ou mais. No Brasil, não existe legislação congénere sustentada na idade de ingresso no Ensino Superior, embora o sistema de cotas, regido pela Lei Estadual n. ${ }^{\circ}$ 5346/2008, contemple situações financeiras desfavoráveis.

A presente investigação pretende caracterizar do ponto de vista sociodemográfico os estudantes M23 de duas IES portuguesas. Os objetivos passam por retratar a autoperceção dos estudantes M23 acerca da sua autoeficácia formativa e o seu envolvimento nos processos de aprendizagem, assim como por explorar a relação entre estas autoperceções, suas dimensões e idade de ingresso no Ensino Superior. 


\section{METODOLOGIA}

\subsection{A MOSTRA}

A amostra foi definida por conveniência e integra 55 sujeitos com idades compreendidas entre os 24 e os 57 anos $(M=34.4 ; D P=9.1)$. É constituída maioritariamente por mulheres, sendo que apenas $40.0 \%$ dos sujeitos são do género masculino. As duas IES de pertença destes estudantes, um instituto politécnico privado e uma universidade privada, ambos do Norte de Portugal, estão equitativamente representadas na amostra $(54.5 \%$ e $45.5 \%$, respetivamente). Cerca de $3 / 4$ da amostra (74\%) frequenta cursos das áreas da Saúde e das Ciências Sociais e Humanas e, em termos de habilitações académicas, ingressou no Ensino Superior com o Ensino Secundário completo.

\subsection{InStRUmentos De AValiação}

O protocolo de recolha de dados integra uma primeira parte relativa a dados sociodemográficos e dois instrumentos adaptados para Portugal: um inventário de envolvimento académico dos estudantes do Ensino Superior (adaptado para Portugal por Marôco, Marôco, Campos, \& Fredricks, 2016) e uma escala de autoeficácia na formação superior (adaptada por Polydoro \& GuerreiroCasanova, 2010).

A autoeficácia traduz-se na crença do estudante relativamente à sua capacidade para organizar e executar as ações necessárias para atingir os objetivos referentes às tarefas académicas no Ensino Superior. A escala é constituída por 20 itens, avaliados numa escala tipo Likert de 6 pontos. Os itens são distribuídos por três dimensões, todas com uma boa consistência interna: (i) "Autoeficácia académica" - confiança na capacidade de aprender, demonstrar e aplicar o conteúdo do curso; (ii) "Autoeficácia na regulação da formação" - confiança do estudante na sua capacidade para estabelecer metas, fazer escolhas, planear, cumprir prazos e autor regular as suas ações no processo de formação; e (iii) "Autoeficácia na interação social” - confiança na capacidade para se relacionar em termos académicos e sociais com os colegas e com os professores. Tal como referem Vieira, Polydoro, e Guerreiro-Casanova (2017), não estão definidas normas para a escala, com base nos estudos de Polydoro, Vieira, e Coimbra (2008) e de Vieira (2010), mas pontuações nas subescalas iguais ou inferiores a 3 indiciam baixos níveis de autoeficácia na respetiva dimensão, e pontuações iguais ou superiores a 5 correspondem a crenças de autoeficácia robustas nas dimensões da escala. As autoras confirmaram o modelo trifatorial da escala, com base em índices de ajustamento satisfatórios.

O Inventário de Envolvimento Académico é constituído por 15 itens, avaliados numa escala de Likert de 5 pontos e organizados em três dimensões: (i) Envolvimento Emocional - reflete as reações positivas e negativas face aos professores, colegas e exigências da escola, nomeadamente em relação ao curso e códigos de conduta, criando laços com a escola e os colegas e vontade para realizar o trabalho escolar exigido; (ii) Envolvimento Cognitivo - investimento e vontade de fazer os esforços necessários para a compreensão e interiorização de ideias complexas e competências com elevado grau de dificuldade; e (iii) Envolvimento Comportamental participação do estudante em atividades académicas, sociais e extracurriculares que acontecem na escola ou que com esta estão relacionados. Marôco et al. (2016), através da análise fatorial confirmatória, sublinham a estrutura trifatorial do instrumento, assim como a sua boa consistência interna: 
O inventário não possui normas, contudo, é possível considerar o ponto ou valor intermédio de cada subescala, situado no valor 3, analisar os resultados em comparação com este valor, podendo estar abaixo ou acima do valor de referência. Assim sendo, os resultados poderão corresponder respetivamente a baixo e elevado Envolvimento Académico para cada uma das subescalas consideradas. (Costa \& Marôco, 2017, p. 40)

Globalmente, o instrumento revela ter boa consistência interna e resultados aceitáveis de validade preditiva, pelo que os autores sugerem a sua utilização na investigação no contexto do Ensino Superior.

\subsection{PROCEDIMENTOS DE RECOLHA E TRATAMENTO DE DADOS}

A recolha de dados foi realizada através de inquérito por questionário, disponibilizado online através do Google Forms no período de março a junho de 2018. Os estudantes foram contactados via e-mail, no sentido de os sensibilizar para a importância da participação no estudo.

Posteriormente, os resultados foram apresentados às IES participantes no estudo, sendo previamente resguardadas as questões éticas relativas ao pedido de autorização e à garantia do anonimato dos sujeitos.

Os dados recolhidos foram submetidos a uma análise quantitativa, com recurso à estatística descritiva e inferencial, através do SPSS - versão 24.0. Foi realizada uma análise descritiva da autoperceção dos estudantes M23 acerca da sua autoeficácia formativa e do seu envolvimento nos processos de aprendizagem, em função da idade de ingresso.

No processo de tratamento de dados, a idade de entrada no Ensino Superior foi operacionalizada em dois grupos (estudantes que ingressaram no Ensino Superior com 26 anos ou menos e estudantes que ingressaram com mais de 26 anos). Esta divisão foi feita posteriormente à análise preliminar dos dados, uma vez que se constataram diferenças em termos do Envolvimento global dos estudantes e, em particular, em termos do Envolvimento Emocional e do Envolvimento Comportamental. As respostas dos grupos foram comparadas através da escala de autoeficácia na formação superior e através do inventário de envolvimento académico dos estudantes do Ensino Superior.

Para avaliar se existiam diferenças estatisticamente significativas entre os dois grupos etários (alunos que entraram no Ensino Superior até aos 26 anos e alunos que entraram no Ensino Superior após os 26 anos), ao nível das pontuações totais das duas escalas, bem como ao nível das diferentes componentes (com exceção do Envolvimento Comportamental e da Autoeficácia na Regulação da Formação), recorreu-se ao teste t-student para amostras independentes, após se ter validado os pressupostos deste método estatístico. Tais pressupostos (normalidade das distribuições e homogeneidade das variâncias) foram avaliados com recurso ao teste de Shapiro-Wilk, para a amostra correspondente aos alunos que entraram no Ensino Superior até aos 26 anos (tendo a normalidade sido assumida para o outro grupo, já que o mesmo tem mais de 30 elementos), e ao teste de Levene, para o grupo de alunos que entrou no Ensino Superior após os 26 anos.

No que diz respeito ao Envolvimento Comportamental e à Autoeficácia na Regulação da Formação, recorreu-se ao teste não paramétrico de Mann-Whitney, dado que os pressupostos do teste $t$-student não eram verificados.

Para estabelecer a relação entre as diversas pontuações (quer os totais das duas escalas, 
quer as de cada componente) e destas com as várias variáveis sociodemográficas, optou-se pela realização da regressão linear.

\section{RES U LTA D O S}

A amostra apresenta-se equilibrada quanto à empregabilidade enquanto razão para o ingresso no Ensino Superior. Em relação a outras razões para a entrada no Ensino Superior, são evocadas, de forma generalizada, as razões relativas ao desenvolvimento pessoal, mas as razões relativas ao estatuto social, interação social e progressão profissional não se mostram importantes.

Quanto às escalas, todos os pressupostos (nomeadamente a normalidade, homogeneidade e independência dos erros) foram validados graficamente. Foram eliminadas variáveis fortemente colineares com as restantes variáveis do modelo, para evitar a existência de multicolinearidade, e, embora se tenham identificado alguns outliers, estas observações não foram eliminadas, uma vez que, após testar o modelo com e sem essas observações, não se verificavam diferenças de relevo.

\subsection{CARACTERIZAÇÃO DO} ENVOLVIMENTO ACADÉmico NOS ESTUDANTES MAIORES DE 23 ANOS

Através da tabela 1 é possível verificar que os estudantes que entram no Ensino Superior pela via dos M23 apresentam, relativamente ao Envolvimento Académico, uma pontuação total bastante acima do valor médio (valor 3), o que revela um Envolvimento Académico globalmente elevado (Costa \& Marôco, 2017, p. 40). O mesmo acontece quando analisamos cada componente individualmente, sendo que os baixos valores do desvio-padrão revelam um bom grau de acordo entre as respostas.

Através da tabela 2 é possível verificar que os estudantes que entram no Ensino Superior pela via dos M23 apresentam, relativamente à Autoeficácia, uma pontuação total acima de 5, o que indicia, de acordo com Vieira et al. (2017), que estes estudantes apresentam, globalmente, crenças de autoeficácia robustas. O mesmo acontece quando analisamos as componentes Autoeficácia na Regulação da Formação e Autoeficácia na Interação Social. No caso da componente Autoeficácia Académica, a pontuação não chega a 5, sendo, no entanto, muito próxima deste valor. Em todos os casos, os baixos valores do desvio-padrão revelam um bom grau de acordo entre as respostas.

TABELA 1

Análises Descritivas para os Índices das Componentes e para a Pontuação Total do Inventário de Envolvimento Académico

\begin{tabular}{lcc}
\hline & Média & Desvio-Padrão \\
\hline Envolvimento Comportamental & 4.47 & 0.46 \\
Envolvimento Emocional & 3.94 & 0.71 \\
Envolvimento Cognitivo & 4.25 & 0.56 \\
Média de todos os itens do Inventário de Envolvimento & 4.22 & 0.42 \\
\hline
\end{tabular}


TA B EL A 2

Análises Descritivas para os Índices das Componentes e para a Pontuação Total da Escala de Autoeficácia na Formação Superior

\begin{tabular}{lcc} 
& Média & Desvio-Padrão \\
\hline Autoeficácia Académica & 4.96 & 0.75 \\
Autoeficácia na Regulação da Formação & 5.25 & 0.75 \\
Autoeficácia na Interação Social & 5.23 & 0.70 \\
Média de todos os itens da Escala da Autoeficácia & 5.14 & 0.67 \\
\hline
\end{tabular}

COMPARAÇÃO DAS PONTUAÇÕES TOTAIS E DE CADA COMPONENTE COM OS GRUPOS ETÁRIOS DE ENTRADA NO ENSINO SUPERIOR

Para avaliar se existem diferenças estatisticamente significativas entre os dois grupos etários anteriormente definidos, ao nível das pontuações totais das duas escalas, bem como ao nível das diferentes componentes (com exceção do Envolvimento Comportamental e da Autoeficácia na Regulação da Formação), recorreu-se ao teste $t$-student para amostras independentes, apenas tendo sido encontradas diferenças estatisticamente significativas para a pontuação total do Envolvimento ( $t(53)$ $=-3.263 ; p=0.002)$ e para o Envolvimento Emocional $(t(53)=-2.561 ; p=0.013)$.

No que diz respeito à pontuação total do Envolvimento, verifica-se que esta é significativamente mais elevada nos estudantes que entraram no Ensino Superior após os 26 anos. De facto, os estudantes que entraram no Ensino Superior pela via dos M23 quando ainda não tinham atingido a idade de 27 anos apresentam uma pontuação total de Envolvimento Académico com média de 3.99 $(D P=0.44)$, enquanto aqueles que já tinham 27 anos ou mais apresentam uma média de $4.35(D P=0.35)$.

De igual forma, no que diz respeito ao Envolvimento Emocional, verifica-se que este é significativamente mais elevado nos estudantes que entraram no Ensino Superior após os 26 anos. De facto, os estudantes que entraram no Ensino Superior pela via dos M23 quando ainda não tinham atingido a idade de 27 anos apresentam uma pontuação total de Envolvimento Emocional com média de 3.63 $(D P=0.73)$, enquanto os que já tinham 27 anos ou mais apresentam uma média de 4.11 $(D P=0.64)$.

Quanto ao Envolvimento Comportamental e à Autoeficácia na Regulação da Formação, recorreu-se ao teste não paramétrico de Mann-Whitney. Verificaram-se diferenças estatisticamente significativas, entre os dois grupos etários, apenas para o Envolvimento Comportamental $(U=229.5 ; z=-2.137 ; p=$ 0.032). De facto, quer a média quer a mediana desta componente são superiores para o grupo de estudantes que entrou no Ensino Superior após os 26 anos, sendo a dispersão dos dados menor para este grupo.

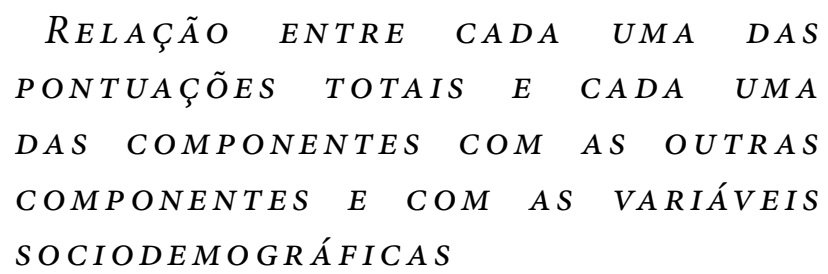

Para estabelecer a relação entre as diversas pontuações (quer as totais, quer as de cada componente) e destas com as várias variáveis sociodemográficas, optou-se pela realização da regressão linear. 
a. Envolvimento Académico como variável dependente:

A regressão linear múltipla permitiu identificar as variáveis Autoeficácia Académica $(\beta=0.621 ; t=6.465 ; p<0.001)$ e Idade de entrada no Ensino Superior $(\beta=$ $0.286 ; t=2.971 ; p=0.004)$ como preditores significativos do Envolvimento Académico, tendo-se obtido um modelo estatisticamente significativo $(F(2,52)=30.317 ; p<0.001)$ que explica $52.1 \%$ da variabilidade da pontuação total do Envolvimento.

\section{b. Envolvimento Comportamental como variável dependente:}

A regressão linear múltipla permitiu identificar as variáveis Autoeficácia na Regulação da Formação $(\beta=0.557 ; t=$ 5.221; $p<0.001)$ e Envolvimento Emocional $(\beta=0.256 ; t=2.400 ; p=0.020)$ como preditores significativos do Envolvimento Comportamental, tendo-se obtido um modelo estatisticamente significativo $(F(2,52)=21.621 ; p<0.001)$ que explica $43.3 \%$ da variabilidade observada na pontuação do Envolvimento Comportamental.

\section{c. Envolvimento Emocional como variável dependente:}

A regressão linear múltipla permitiu identificar as variáveis Autoeficácia Académica $(\beta=0.372 ; t=3.023 ; p=0.004)$ e Idade de entrada no Ensino Superior $(\beta=$ $0.258 ; t=2.096 ; p=0.041)$ como preditores significativos do Envolvimento Emocional, tendo-se obtido um modelo estatisticamente significativo $(F(2,52)=8.353 ; p=0.001)$ mas que explica uma percentagem mais baixa da variabilidade da variável dependente, face aos modelos anteriores (21.4\%).

\footnotetext{
d. Envolvimento Cognitivo como variável dependente:
}

A regressão linear permitiu identificar a variável Autoeficácia Académica $(\beta=$ 0.487; $t=4.059 ; p<0.001)$ como preditora significativa do Envolvimento Cognitivo, tendo-se obtido um modelo estatisticamente significativo $(F(1,53)=16.477 ; p<0.001)$ mas que, tal como o modelo anterior, explica uma percentagem mais baixa da variabilidade da variável dependente, face aos dois primeiros modelos (22.3\%).

e. Autoeficácia como variável dependente: A regressão linear múltipla permitiu identificar as variáveis Envolvimento Cognitivo $(\beta=0.273 ; t=2.565 ; p=0.013)$

e Envolvimento Comportamental $(\beta=$ $0.558 ; t=5.244 ; p<0.001)$ como preditores significativos da pontuação total da Autoeficácia, tendo-se obtido um modelo estatisticamente significativo $(F(2,52)$ $=27.338 ; p<0.001)$ que explica $49.4 \%$ $\mathrm{da}$ variabilidade $\mathrm{da}$ pontuação total $\mathrm{da}$ Autoeficácia.

f. Autoeficácia Académica como variável dependente:

A regressão linear múltipla permitiu identificar as variáveis Autoeficácia na Interação Social $(\beta=0.393 ; t=3.289 ; p=0.002)$ e Autoeficácia na Regulação da Formação $(\beta$ $=0.482 ; t=4.030 ; p<0.001)$ como preditores significativos da Autoeficácia Académica, tendo-se obtido um modelo estatisticamente significativo $(F(2,52)=52.363 ; p<0.001)$ que explica $65.5 \%$ da variabilidade da pontuação da Autoeficácia Académica.

g. Autoeficácia na Regulação da Formação como variável dependente:

A regressão linear múltipla permitiu identificar as variáveis Autoeficácia na Interação Social $(\beta=0.373 ; t=3.040 ; p$ $=0.004)$ e Autoeficácia Académica $(\beta=$ 
0.494; $t=4.030 ; p<0.001)$ como preditores significativos da Autoeficácia na Regulação da Formação, tendo-se obtido um modelo estatisticamente significativo $(F(2,52)=$ 50.399; $p<0.001)$ que explica $64.7 \%$ da variabilidade da pontuação da Autoeficácia na Regulação da Formação.

h. Autoeficácia na Interação Social como variável dependente:

A regressão linear permitiu identificar a variável Autoeficácia na Regulação da Formação $(\beta=0.744 ; t=8.104 ; p<0.001)$ como preditor significativo da Autoeficácia na Interação Social, tendo-se obtido um modelo estatisticamente significativo $(F(1,53)=65.679 ; p<0.001)$ que explica $54.5 \%$ da variabilidade da pontuação da Autoeficácia na Interação Social.

Em síntese, e face aos objetivos enunciados quanto à autoperceção dos estudantes M23 acerca da sua autoeficácia formativa e do seu envolvimento nos processos de aprendizagem, os resultados apontam para um elevado envolvimento académico e para crenças robustas de autoeficácia. Verifica-se, ainda, que existem diferenças significativas entre os dois grupos definidos após a exploração inicial dos dados (até aos 26 anos e com mais de 26 anos) quanto ao envolvimento académico global e, em particular, no que diz respeito às dimensões emocional e comportamental.

No que concerne à relação entre as autoperceções, suas dimensões e idade de ingresso noEnsinoSuperior destes estudantes, é de realçar que, em todas as análises de regressão anteriormente mencionadas, se verifica um impacto positivo dos preditores nas variáveis dependentes consideradas.

\section{DISCUSSÃO E CONSIDERAÇÕES F I N A I S}

Os estudantes que entram no Ensino Superior pela via dos M23 evidenciam um bom envolvimento académico, mostram-se confiantes na sua capacidade para ter sucesso nas tarefas que decorrem da frequência do Ensino Superior e evocam a empregabilidade como principal razão para o ingresso, seguida do desenvolvimento pessoal. Os resultados permitiram a definição de dois grupos distintos em função da idade: até aos 26 anos e com mais de 26 anos.

Os estudantes que ingressaram com mais de 26 anos destacam-se pelo maior envolvimento académico global e, em particular, pelo maior envolvimento emocional e comportamental. Isto significa que os estudantes mais velhos estabelecem um maior compromisso e uma ligação mais forte com o Ensino Superior, o que pode relacionar-se com níveis mais elevados de maturidade e consciência psicossocial. $\mathrm{O}$ envolvimento académico, ao implicar um estado afetivo-cognitivo positivo, aproxima-se do conceito de experiência ótima preconizado por Csikszentmihalyi (1990), dominado pela satisfação intrínseca e caracterizado pela perda de autoconsciência, distorção da perceção do tempo, atenção focalizada e união entre corpo e mente. Este tipo de experiências concretiza-se num equilíbrio entre os desafios das atividades académicas e as competências que os sujeitos julgam ter para a sua realização bem-sucedida, promovendo a persistência nas tarefas.

Para além da idade de entrada no Ensino Superior, o Envolvimento Académico e, em particular, o Envolvimento Emocional, podem ser previstos pela Autoeficácia Académica, entendida como a confiança na capacidade de aprender, demonstrar e aplicar o conteúdo do curso. A Autoeficácia global depende do Envolvimento Cognitivo e do Envolvimento 
Comportamental. No entanto, a Autoeficácia Académica mostra-se fraca preditora do Envolvimento Cognitivo, mas consegue prever, quer o Envolvimento Académico, quer o Emocional. Esta análise permite-nos depreender que a Autoeficácia Académica é uma variável central quando se analisam os estudantes que ingressam pela via dos M23.

A participação do estudante em atividades académicas, sociais e extracurriculares que acontecem na escola ou que com esta estão relacionadas (Envolvimento Comportamental) pode ser prevista pela Autoeficácia na Regulação da Formação (ou seja, pela confiança do estudante na sua capacidade para estabelecer metas, fazer escolhas, planear, cumprir prazos e autorregular as suas ações no processo de formação), e pelo Envolvimento Emocional (reações face aos professores, colegas e exigências da escola, nomeadamente em relação ao curso e códigos de conduta, laços com a escola e colegas e motivação para a realização escolar). Assim, estudantes mais confiantes nas suas competências de planeamento, gestão e autorregulação são estudantes mais participativos nas atividades académicas e outras, e demonstram uma atitude mais positiva face aos contextos académicos, suas características, dinâmicas, exigências e agentes sociais.

A divisão em dois grupos (até aos 26 e maiores de 26 anos) vai ao encontro dos contributos de Arnett (2000), quando evidencia a existência de períodos distintos na idade adulta. Esta divisão é congruente também com a Teoria das Estações da Vida Adulta de Levinson (1977), ainda que as idades cronológicas possam apresentar alguma variabilidade na definição das diferentes fases. Contudo, as razões para o ingresso no Ensino Superior não diferem quando é tomada em consideração a idade, pelo que a empregabilidade parece continuar a justificar o acesso ao Ensino Superior, mesmo no caso dos estudantes mais velhos, sublinhandose assim a função instrumental da formação superior. Esta tendência é consonante com as questões globais que marcam a atualidade, nas quais a competitividade e as lógicas de mercado se sobrepõem às preocupações psicossociais relacionadas com o desenvolvimento integral dos sujeitos (Barros, 2011, 2013).

O nosso estudo permite concluir que os 26 anos constituem um marco importante quando o objeto de estudo são os estudantes M23. As políticas de educação no que diz respeito ao acesso ao Ensino Superior pautamse pela diversidade. A idade é, nalguns casos, sobretudo na Europa, um critério para a possibilidade de acesso e ingresso através de um contingente especial. Todavia, também nestes casos, a idade é variável, desde os 21 anos, como no Reino Unido, até aos 25, como é o exemplo espanhol. Em qualquer dos casos, os estudantes mais velhos e, portanto, mais maduros, tendo adiado a formação superior, ou tendo-a equacionado pela primeira vez de uma forma mais sustentada, distinguem-se dos colegas mais jovens, mesmo daqueles que ingressaram no Ensino Superior pela mesma via.

Ainda que a empregabilidade, como acima referido, esteja relacionada com as razões do ingresso no Ensino Superior, o sucesso destes estudantes em termos de eficácia formativa, envolvimento e adaptação ao meio académico foram sistematizadas por Silva e Nascimento (2012):

(a) a experiência de vida e a prática profissional diária que auxiliam à compreensão dos aspetos teóricos lecionados e potenciam a mobilização de diversas competências para o contexto académico; (b) o apoio familiar, de outros significativos, da entidade patronal e dos atores institucionais - docentes, funcionários e colegas - ; (c) a participação ativa nas iniciativas 
da faculdade; (d) a possibilidade de articulação/ flexibilidade de horários/exigências escolares com as responsabilidades profissionais; (e) as capacidades cognitivas e o domínio das tecnologias para acesso à informação; (f) o acompanhamento próximo e sistemático por parte dos docentes; e (g) a existência de uma articulação positiva entre o domínio académico e as restantes áreas de vida, pessoais e profissionais. (p. 45)

Se, por um lado, os estudos apontam para múltiplas motivações associadas ao acesso, para uma experiência positiva de integração e para sugestões a implementar pelas Instituições de Ensino Superior no sentido de promover o sucesso destes estudantes, há, por outro lado, a perceção de que o regime de ingresso via M23 anos beneficia sobretudo os adultos mais novos e mais qualificados, colocando reticências em relação ao seu verdadeiro propósito, ou seja, a diversificação dos públicos a frequentar o Ensino Superior (Pires, 2008). O nosso estudo, à semelhança do preconizado por Pires (2008), mostra que, para além da caracterização dos novos públicos do Ensino Superior, torna-se imprescindível conhecer as suas dinâmicas motivacionais e o seu envolvimento na formação, o que passa pela identificação das lógicas subjacentes à procura e realização de novas aprendizagens.

O carácter exploratório deste estudo encerra algumas limitações, que se prendem, essencialmente, com a dimensão da amostra. Sugerem-se outros estudos, com uma amostra superior a 100 sujeitos, no sentido de se poder contrastar os seus resultados com os que agora se apresentam e, assim, sustentar a generalização de resultados.

\section{REFERÊ NCIAS}

Almeida, A., Quintas, H., \& Gonçalves, T. (2016). Estudantes não-tradicionais no Ensino Superior: Barreiras à aprendizagem e na inserção profissional. Laplage em Revista, 2(1), 97-111.

Arnett, J. (2000). Emerging adulthood: A theory of development from the late teens through the twenties. American Psychologist, 55(5), 469-480.

Baptista, A., Bessa, J., \& Tavares, J. (2009). Motivação para o ingresso e aprendizagem no Ensino Superior: O caso dos estudantes maiores de 23 da Universidade de Aveiro. In M. Silva (Coord.), Proceedings of the 10th Luso-Afro-Brazilian Conference of Social Sciences "Sociedades desiguais e paradigmas em confronto" (pp. 163-173). Braga: Universidade do Minho.

Barros, R. (2011). Genealogia dos conceitos em educação de adultos: Da educação permanente à aprendizagem ao longo da vida - Um estudo sobre os fundamentos políticopedagógicos da prática educacional. Lisboa: Chiado Editora.

Barros, R. (2013). Educação de adultos. Conceitos, processos e marcos históricos. $\mathrm{Da}$ globalização ao contexto português. Lisboa: Editora Piaget.

Brás, J. V., Jezine, E., Fonseca, S., \& Gonçalves, M. N. (2012). A universidade portuguesa: O abrir do fecho de acesso - O caso dos maiores de 23 anos. Revista Lusófona de Educação, 21, 163-178.

Costa, A., \& Marôco, J. (2017). Inventário de Envolvimento Académico dos Estudantes do Ensino Superior. In L. S. Almeida, M. R. Simões, \& M. M. Gonçalves (Coords.), Adaptação, desenvolvimento e sucesso académico dos estudantes do Ensino Superior: Instrumentos de avaliação (pp. 33-44). Braga: ADIPSIEDUC (Associação 
para o Desenvolvimento da Investigação em Psicologia da Educação).

Csikszentmihalyi, M. (1990). Flow: The psychology of optimal experience. Journal of Leisure Research, 24(1), 93-94.

Davies, P., Osborne, M., \& Williams, J. (2002). For me or not for me? - That is the question. A study of mature students decision making and higher education. Consultado em fevereiro 20, 2018, em https://dera.ioe.ac.uk//4675/

Direção-Geral do Ensino Superior. (2018). Estudos e estatísticas. Consultado em outubro 30, 2018, em https://www.dges.gov. pt/pt/pagina/estudos-e-estatisticas

Engrácia, P., \& Baptista, J. (2018). Percursos no Ensino Superior: Situação após quatro anos dos alunos inscritos em licenciaturas de três anos. Lisboa: Direção-Geral de Estatísticas da Educação e Ciência (DGEEC).

Levinson, D. (1977). The seasons of a man's life. New York: Alfred A. Knoff.

Marôco, J. P., Marôco, A. L., Campos, J. A., \& Fredricks, J. A. (2016). University student's engagement: Development of the university student engagement inventory (USEI). Psicologia: Reflexão e Crítica, 29, 1-12. doi:10.1186/s41155-016-0042-8

Monteiro, A., Barros, R., \& Moreira, J. A. (2015). Novos públicos do Ensino Superior: Abordagem à aprendizagem de estudantes maiores de 23 anos. Revista Portuguesa de Pedagogia, 49(1), 131-149. doi:10.14195/164786144916

Pereira, E. (2008). Alunos maiores de 23 anos: Motivações para o ingresso no Ensino Superior na UP (Dissertação de mestrado). Faculdade de Letras da Universidade do Porto, Porto, Portugal.

Pires, A. L. (2008, dezembro). Novos públicos no Ensino Superior em Portugal. Contributos para uma problematização. Comunicação apresentada no III Seminário LusoBrasileiro: Trabalho, Educação e Movimentos
Sociais, Faculdade de Psicologia e Ciências da Educação, Universidade de Lisboa, Lisboa, Portugal.

Polydoro, S. A. J., \& Guerreiro-Casanova, D. C. (2010). Escala de autoeficácia na formação superior: Construção e estudo de validação. Avaliação Psicológica, 9(2), 267-278.

Polydoro, S. A. J., Vieira, D., \& Coimbra, J. L. (2008, outubro). Adaptação para a realidade portuguesa da escala Auto-Eficácia na Formação Superior (AEFS). Comunicação apresentada no XIII Congresso de Avaliação Psicológica: Formas e contextos, Universidade do Minho, Braga, Portugal.

Silva, R., \& Nascimento, I. (2010). Os estudantes maiores de 23 anos no Ensino Superior português. Estudo crítico e revisão documental. Revista Brasileira de Orientação Profissional, 11(1), 73-82.

Silva, R., \& Nascimento, I. (2012). Estudantes maiores de 23 anos em Psicologia: Motivações e processo de integração. Revista Brasileira de Orientação Profissional, 13(1), 37-47.

Soares, D., Almeida, L., \& Ferreira, J. (2010). Percursos vocacionais e vivências académicas: $\mathrm{O}$ caso dos alunos maiores de 23 anos. Psicologia, Educação e Cultura, $X I V(1), 203-214$.

Soares, J. (2016). A integração do estudante adulto na universidade de Lisboa. A transição dos maiores de 23 para o Ensino Superior: Potencialidades e dificuldades sentidas. In J. Marques, M. Martins, C. Doutor, \& T. Gonçalves (Eds.), Univers(al) idade. Estudantes «não tradicionais» no Ensino Superior: Transições, obstáculos $e$ conquistas (pp. 35-42). Consultado em março 6, 2019, em shorturl.at/ciT29

Ventura, T. (2009). Estudio sobre los mayores de 23 años en la universidad moderna de Lisboa. Un enfoque metodológico autoevaluativo (Tese de doctorado). Universidad de Sevilla, Sevilla, España. 
Vieira, D. A. (2010). Auto-eficácia na formação superior: Um factor protector face ao insucesso académico? In A. S. Pereira, H. Castanheira, A. C. Melo, A. I. Ferreira, \& P. Vagos (Eds.), Apoio psicológico no Ensino Superior: Modelos e práticas (pp. 355-361). Aveiro: Universidade de Aveiro/RESAPESAP.

Vieira, D. A., Polydoro, S. A. J., \& GuerreiroCasanova, D. (2017). Autoeficácia na formação superior (AEFS). In L. S. Almeida, M. R. Simões, \& M. M. Gonçalves (Coords.), Adaptação, desenvolvimento e sucesso académico dos estudantes do Ensino Superior: Instrumentos de avaliação (pp. 111-123). Braga: ADIPSIEDUC (Associação para o Desenvolvimento da Investigação em Psicologia da Educação).

\section{LEG ISLAÇ Ã O CONSULTADA}

Decreto-Lei no 64/2006, de 21 de março. Regulamenta as provas especialmente adequadas destinadas a avaliar a capacidade para a frequência do ensino superior dos maiores de 23 anos, previstas no n. ${ }^{\circ} 5$ do artigo $12 .^{\circ}$ da Lei n. ${ }^{\circ} 46 / 86$, de 14 de Outubro (Lei de Bases do Sistema Educativo). Disponível em https://dre.pt/application/file/a/667872

\section{FORMATIVE SELF-EFFICACY AND INVOLVEMENT IN THE LEARNING PROCESSES OF PORTUGUESESTUDENTS OLDER THAN 23 YEARS}

\section{Abstract}

The approval of the special access to Higher Education policy for mature students, over 23 years old, is a main challenge faced by Higher Education Institutions (HEIs), that is, the need to construct educational responses taking into account the heterogeneity of its audience. The present exploratory study aims to portray the self-perception of these students about their formative self-efficacy and their academic involvement, according to the age of admission, and explore the relationship between these self-perceptions, their dimensions and the age of entry into Higher Education. Data were collected from 55 students from two private HEIs, through a questionnaire survey. Its results were treated by descriptive and linear regression analysis and show that it is possible to differentiate two groups of students according to their age, up to the age of 26 and over, the latter being characterized by greater academic involvement and greater self-perception of formative effectiveness. The discussion of these results, in light of the theoretical frameworks for the development of adulthood, is of particular relevance for the promotion of equity of access and entry into Higher Education.

KEYWORDs: Higher Education; Formative selfefficacy; Academic involvement 
AUTOEFICACIA FORMATIVA Y PARTICIPACIÓN EN LOS PROCESOS DE APRENDIZAJE DE LOS ESTUDIANTES PORTUGUESES MAYORES DE 23 AÑOS

\section{RESUMEN}

La aprobación del régimen de acceso a la Enseñanza Superior por la vía de los "mayores de 23 ” es uno de los principales desafíos con que se enfrentan las Instituciones de Educación Superior (IES), es decir, la necesidad de construir respuestas educativas teniendo en cuenta la heterogeneidad de su público. El estudio aquí presentado tiene por objetivo retratar la autopercepción de estos estudiantes acerca de su autoeficacia formativa y su implicación académica, en función de la edad de ingreso. Se recogieron datos de 55 estudiantes de dos IES privados, a través de encuestas por cuestionarios, los cuales fueron sujetos a análisis descriptivos y de regresión lineal. Los resultados muestran que es posible diferenciar dos grupos de estudiantes en función de la edad, hasta los 26 años y más, siendo que este último se destaca por el mayor compromiso académico y por la mayor autopercepción de eficacia formativa. La discusión de estos resultados, a la luz de los referenciales teóricos del desarrollo de la edad adulta, asume particular relevancia para la promoción de la equidad de acceso e ingreso en la Enseñanza Superior.

Palabras clave: Enseñanza Superior; Autoeficacia formativa; Participación académica

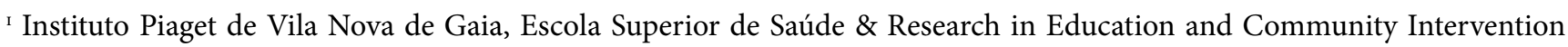
(RECI), Portugal. ORCID: 0000-0002-3596-9992

II Universidade do Porto, Faculdade de Psicologia e de Ciências da Educação \& Centro de Investigação e Intervenção Educativas (CIIE), Portugal. ORCID: 0000-0002-1369-3462

III Universidade Lusófona do Porto, Faculdade de Psicologia, Educação e Desporto \& Research in Education and Community Intervention (RECI), Portugal. ORCID: 0000-0003-4658-9781
} 